\title{
Angioplasty Using Drug-coated Balloons in High-grade Ostial Vertebral Artery Stenosis: First Experiences in a Small Case Series
}

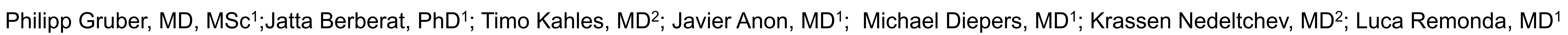

1 Department of Neuroradiology, Cantonal Hospital of Aarau, Aarau, Switzerland

2 Department of Neurologie, Cantonal Hospital of Aarau, Aarau, Switzerland

\section{Background}

Approximately $20-25 \%$ of ischemic strokes occur in the posterior circulation; and $10-20 \%$ of the patients with ostial vertebral artery stenosis (OVAS) will suffer from a stroke ${ }^{1}$. There is an ongoing debate on the treatment modalities for OVAS patients whether patients benefit from endovascular or from best-medical treatment alone since the VIST, VAST and CAVATS trial. Today, best medical treatment using antiplatelet agents is considered first-line treatment of OVAS. However, endovascular OVAS treatment might be considered especially in patients with hemodynamic vertebrobasilar insufficency, bilateral $>70 \%$ vertebral artery stenosis (VAS) and in patients with unilateral VAS with contralateral hypoplastic or occluded vessels. Initial good clinical results and high success rates have been reported for percutaneous angioplasty with or without stenting. However, the re-stenosis rate was reported as high as $10-67 \%$.

During the last decade, drug-eluting stents (DES) and drug-coated balloons (DCB) have been established in the field of interventional cardiology with convincing short- and long-term results. DES in patients with high grade OVAS appear to have lower re-stenosis rates compared to the previously used bare-metal stents (BMS). Data on treatment of high-grade OVAS with DCB is scarce.

\section{Purpose}

In this case series we assessed feasibility and safety of DCB-PTA in patients with high-grade OVAS using Neuro Elutax SV (Aachen Resonance, Aachen, Germany) and SeQuent Please NEO (B.Braun, Melsungen, Germany) .
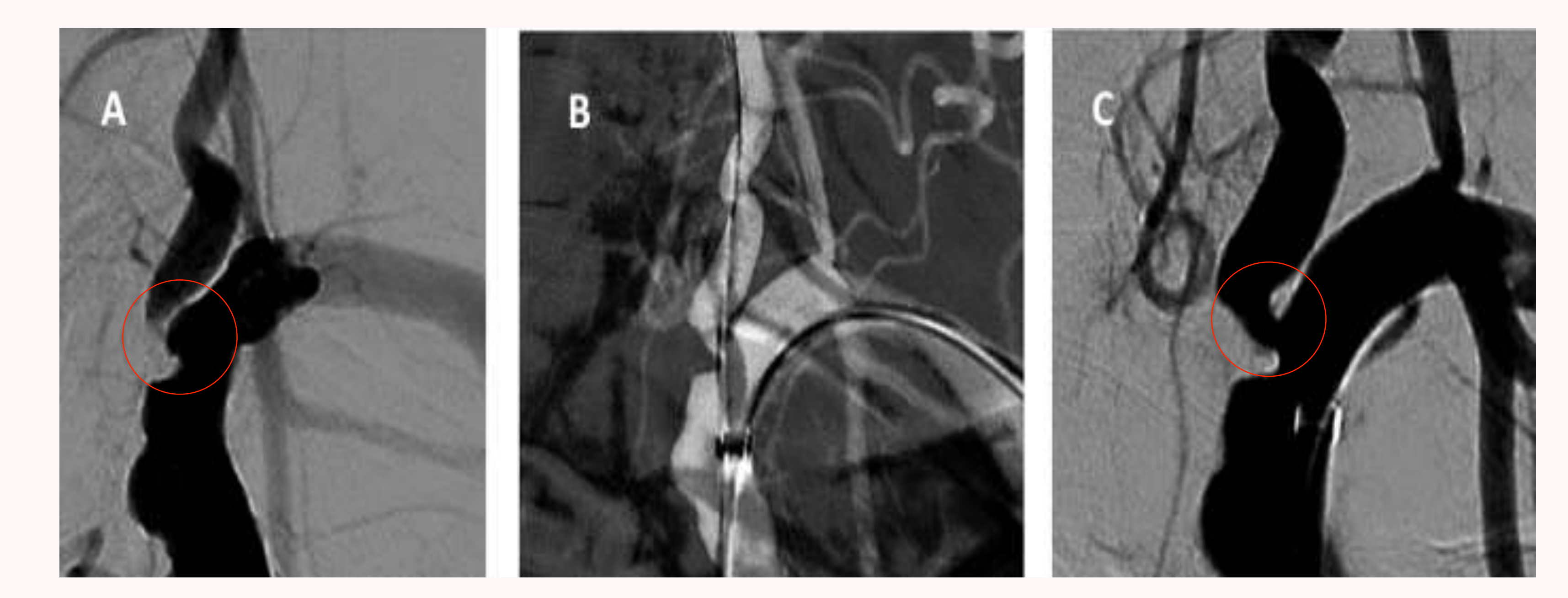

Patient with known extensive atherosclerotic arteriopathy of supra-aortic arteries:
A) Pre-procedural angiogram of a high-grade, excentric OVAS of the left vertebral artey.

B) Intra-procedural inflated angioplasty balloon (SeQuent Please NEO). 4-times angioplasty with 2 times $10 \mathrm{~mm} \times 2 \mathrm{~mm}$ SeQuentPlease NE0 and 2 times with a $10 \mathrm{~mm} \times 3 \mathrm{~mm}$ SeQuent Please NEO. Please NEO and 2 times with a $10 \mathrm{~mm} \times 3 \mathrm{~mm}$ SeQuent Please NEO.
C) Post-procedural angiogram with residual stenosis $(50 \%)$ with good restoration of ante-grade flow and partial regress of ipsi-lateral
occipito-vertebral anastomoses.

\section{Material and Methods}

Retrospective, mono-center case series of six patients with high-grade ostial vertebral artery stenosis treated with PTA using a drug-coated balloon.

Outcome measurement were post-procedural angiographic stenosis degree according to the VOTE method criteria, as well as the post-procedural shortterm (within 24hours) and long-term ultra-sonographic stenosis degree. Additionally, all peri-procedural complications as well as clinical follow-up (mRS) were assessed.
Results

\begin{tabular}{|c|c|}
\hline Clinical Characteristics & $\mathrm{N}=6$ \\
\hline Sex (male) - no. $(\%)$ & $3(50 \%)$ \\
\hline Age in years - median (IQR) & $72(66-76)$ \\
\hline Hypertension - no. (\%) & $6(100 \%)$ \\
\hline Atrial Fibrillation - no. (\%) & $3(50 \%)$ \\
\hline Dyslipidemia - no. (\%) & $4(64 \%)$ \\
\hline Nicotine abuse - no. (\%) & $5(83 \%)$ \\
\hline Diabetes mellitus - no. (\%) & $0(0 \%)$ \\
\hline Kidney failure - no. (\%) & $7(32)$ \\
\hline NIHSS on admission - median (IQR) & $0(0-0)$ \\
\hline Most common clinical symptom: vertigo/ dizziness & $4(67 \%)$ \\
\hline \multicolumn{2}{|l|}{ IQR, interquartile range; no., number; NIHHS, National Institute of Health Stroke Scale; } \\
\hline Lesion Characteristics & $\mathrm{N}=6$ \\
\hline Lesion side (left) - no. (\%) & $6(100 \%)$ \\
\hline Pre-interventional stenosis degree VOTE in perecentage - median (IQR) & $73 \%(60-80)$ \\
\hline Lesion length in mm - median (IQR) & $6 \mathrm{~mm}(4-13)$ \\
\hline \multicolumn{2}{|l|}{ Contralateral vertebral artery (VA) } \\
\hline Hypoplastic V4-Segment of the VA - no. (\%) & $2(34 \%)$ \\
\hline Occlusion/ Pseudoocclusion of the VA- no. (\%) & $2(34 \%)$ \\
\hline High-grade Stenosis- no. (\%) & $1(17 \%)$ \\
\hline Moderate Stenosis- no. $(\leq 50 \%)$ & $1(17)$ \\
\hline
\end{tabular}

\begin{tabular}{l|c}
\hline Procedure Characteristics & N=6 \\
\hline General Anesthesia - no. (\%) & $4(67 \%)$ \\
\hline Neuro Elutax SV as first DCB - no. (\%) & $5(83 \%)$ \\
\hline SeQuent Please NEO as first DCB - no. (\%) & $1(17 \%)$ \\
\hline Change to another DCB - no. (\%) & $1(17 \%)$ \\
\hline Second larger-size DCB use - no. (\%) & $4(67 \%)$ \\
\hline DCB, drug-coated balloon; no., number & \\
\hline Outcome Measures & N=6 \\
\hline Post-interventional stenosis degree VOTE in perecentage - median (IQR) & $45 \%(25-50)$ \\
\hline Mean Follow-up period in months & 5.3 \\
\hline Recurrent clinical ischemic event - no. & 0 \\
\hline Re-stenosis rate at follow-up - no. & 0 \\
\hline Major peri-procedural complicaions - no. & 0 \\
\hline Dissection - no. & 0 \\
\hline Vessel perforation - no. & 0 \\
\hline Hemorrhage - no. & 0 \\
\hline Distal Ischemic Event - no. & 0 \\
\hline Mortality - no. & 0 \\
\hline IQR, interquartile range; no., number; VotE, vertebral origin treatment with endovascular method &
\end{tabular}

\section{Discussion and Conclusion}

Our preliminary results demonstrated that OVAS treatment with DCB in appropriately selected patients is feasible, safe and revealed sustained short-outcome results. These findings are in line with a previous case report of DCB in OVAS ${ }^{2}$. Promising results have been shown for DCB treatment in symptomatic intracranial atherosclerotic disease (ICAD). These results might even encourage the use of $D C B$ also in the extracranial vasculature ${ }^{3,4}$.

Thus, DCB-PTA might be considered as a novel treatment option in patients with ostial vertebral artery stenosis. 